\title{
Preexposure pointing frequency effects on adaptation to prismatic viewing*
}

\author{
BENJAMIN WALLACE; \\ Kent State Liniversity, Kent, Ohio 44242
}

\begin{abstract}
The effect of frequency of preexposure pointing was studied to determine its effect on subsequent adaptation to a prismatic exposure. Results showed that when $S$ s pointed equally to each target in a preexposure pointing task. adaptation was found to be greatest for the target on S's prismatically shifted visual field periphery. However, when Ss pointed more frequently in the preexposure condition to a more central target. adaptation was greatest for this target.
\end{abstract}

Typically, studies in visual adaptation require Ss to perform both a preexposure and postexposure test of target pointing accuracy under normal vision. The degree of adaptation acinieved by the intervening exposure to a prismatically displaced visual field is defined by the degree to which the postexposure measurements, relative to the preexposure. are biased in the direction opposite to that of the optical displacement ("negative aftereffect").

Recently, Wallace (1972) and Melamed, Halay, and Gildow (1973) have shown differential adaptation for targets as a function of where targets are located in the visual field. Wallace (1972) found that the greatest adaptation was seen for targets in the peripheral visual field. Thus, when observable targets were prismatically shifted to the right part of S's visual field, the greatest adaptation was found for the target located on the right edge of the visual field. When targets in the visual field were prismatically shifted to the left of normal, straight-ahead viewing, the greatest adaptation was found for the left, most peripheral target. This phenomenon was replicated by Melamed, Halay, and Gildow (1973).

Since adaptation for peripheral targets is greater in magnitude than for more centrally located targets, this could be viewed as indicating that more information must be available to $S$ in detecting the discrepancy between the actual location of peripheral targets in the pre- and postexposure conditions and their apparent location during prismatic viewing. When $\mathrm{S}$ views stimuli through prisms, several sources of information are available to help detect the discrepancy between the viewed and actual location of these stimuli. According to Harris (1965), adaptation to prismatic viewing results from proprioceptive feedback which $S$ receives concerning the location of his arm with respect to other

*Based in part upon a dissertation submitted in partial fulfillment of the $\mathrm{PhD}$ degree requirement at Kent State University. Much appreciation is expressed to Lawrence $\mathrm{E}$. Melamed for his aid and counsel during the course of the research.

†Reprint requests should be sent to Benjamin Wallace, Department of Psychology, Western Illinois University, Macomb, Illinois 61455 . parts of his body during an adaptation procedure. In addition, Rock (1966) has suggested that memory components from preexposure to exposure viewing of the location of S's arm and other stimuli in the visual field might account for aspects of adaptation to a prismatic displacement. Thus, a person who sees his arm through prisms experiences a discrepancy between visual and proprioceptive feedback concerning arm location and between the memory of preexposure, proprioceptive feedback, and the present proprioceptive feedback concerning arm location. When he corrects arm movements according to the perceived discrepancies, the S's responses to his own proprioceptive feedback are altered such that the preexposure movements are biased in the direction opposite to that of the optical displacement. Since S moves his arm further in pointing to a target in the periphery of his prismatically shifted visual field than in pointing to more centrally located targets, the perceived discrepancy between preexposure and exposure movements must be greater for the peripheral than for the central targets. Thus, due to the greater perceived discrepancy, the proprioceptive feedback will undergo even greater adaptation, as measured by the overcompensation during the postexposure test.

This explanation for visual adaptation was further extended by the present study. This study proposed that proprioceptive feedback from possible memory of arm location with respect to target location and body position could be altered by requiring $S$ to point a different number of times to the different targets in the visual array during the preexposure condition. It was felt that the independently varied magnitude of proprioceptive feedback, along with target placement, would affect the degree of adaptation in the following manner. If $S$ were required to point more often to, say, a target more centrally located during prism exposure than the other targets, there would be more proprioceptive information available concerning the location of this target with respect to body position from the preexposure procedures. Thus, during prism exposure, since $S$ was more certain of or had a better memory for the preexposure proprioceptive feedback of pointing to the now most centrally located target, he would be more 


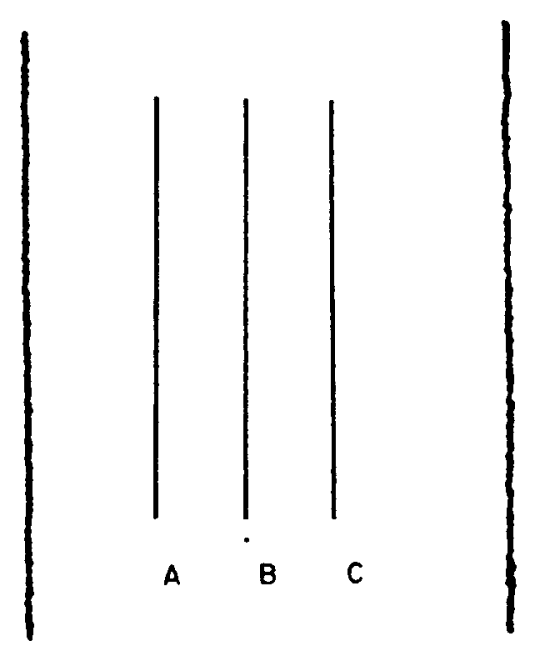

\section{EDGES OF VISUAL FIELD}

\section{NORMAL VIEWING, NO PRISM SHIFT}

Fig. 1. S's view of a $33-\mathrm{cm}$-wide visual field with three targets present under normal vision. All targets are symmetrically located with respect to body position.

aware of or certain of the discrepancy between preexposure and exposure proprioception. Since this discrepancy is one of the believed sources for production of the negative aftereffect, increasing the discrepancy should increase the effect. For example, if three targets were present in the visual field and $\mathrm{S}$ wore base right prisms where the visual field is shifted to the left of normal, straight-ahead viewing, the greatest adaptation would normally be expected for the left target which was prismatically shifted to the periphery of the visual field. Due to this prismatic shifting and because of the greater amplitude of arm movement to that target during prism exposure, greatest adaptation occurs for the most peripherally located target. However, if $E$ now arranges that $\mathrm{S}$ point more often to the right target during the preexposure condition, which when prismatically shifted will be the most centrally located target, then greater adaptation might be produced for this target than for the other two targets because of the greater magnitude of the preexposure proprioceptive feedback leading to a greater perceived discrepancy during prism exposure. If a memory component, in this case for proprioceptive location of S's arm as a function of preexposure pointing frequency, does affect magnitude of visual adaptation for specific targets, this would show the importance of proprioceptive feedback concerning arm location not only during prism exposure. but also during the preexposure period. The present study considers this possibility and the role of preexposure pointing frequency in the study of visual adaptation.

\section{METHOD}

\section{Subjects}

Forty-eight right-handed female undergraduates from the introductory psychology sections served as Ss. Only Ss who did not wear corrective glasses were selected.

\section{Apparatus}

Ss were seated at a small wooden table with their heads securely positioned on a combination head- and chinrest. Throughout all experimental conditions, Ss wore Risley rotating prisms at tached to the front surface of welder's goggles. In front of $\mathrm{S}$, on the table, was a rectangular wooden box, $49.5 \mathrm{x}$ $77.5 \mathrm{~cm}$, open on the side facing $\mathrm{S}$. When $\mathrm{S}$ was asked to observe movements of her hand. it was placed in an aluminum holder running on an aluminum track on the top level of the wooden box. Immediately behind the track was a wooden backboard that extended the entire width of the box. A tagboard with three unnumbered targets was placed over the backboard for the entire experiment. The targets consisted of three black lines, $0.4 \mathrm{x}$ $15.3 \mathrm{~cm}$ and $5.1 \mathrm{~cm}$ apart centered on the tagboard. Target position during preexposure and exposure conditions are illustrated in Figs. 1.2. and 3. During the pre- and postexposure
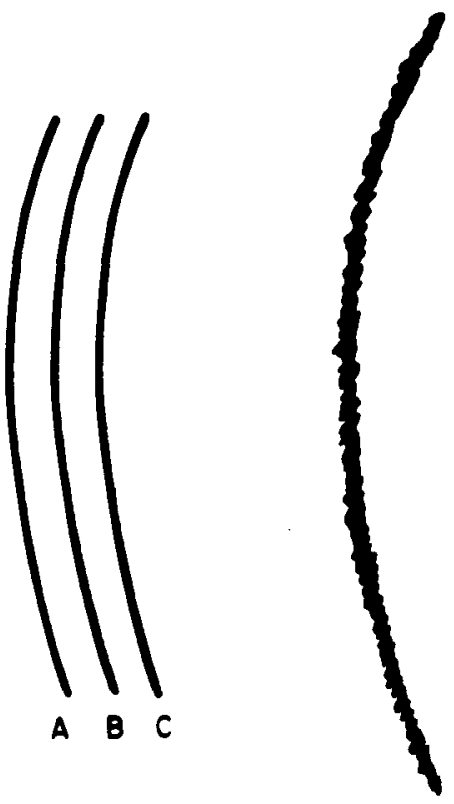

\section{EDGES OF VISUAL FIELD \\ BASE RIGHT PRISM SHIFT}

Fig. 2. S's view of a 33-cm-wide visual field with three visual targets present during 20-diopter prismatic displacement, base right. Target $A$ forms the boundary of the visual field on the left and is, thus, most peripherally located with respect to body position. Target $C$ is the extreme nonboundary target and is most centrally located with respect to body position during prism exposure. 
tests, S's hand was kept out of sight by placing it in a holder on an aluminum slide in the lower compartment (interior) of the box. The slide was found directly below the one on the upper level of the box. The location of S's judgment of a target line was determined by reading a value from a measuring stick that was attached to the interior slide and, thus, moved with it.

\section{Procedure}

All Ss underwent a pointing accuracy task before and after a 10-min exposure to 20-diopter lateral prismatic displacement. The Risley prisms were set at 0 diopter for the pre- and postexposure pointing tasks. In the preexposure portion of the experiment, half of the $S s$ in the experimental group were required to point with their right hands 29 times to the target that. after the prismatic shift, would be the one most centrally located, while pointing only five times to each of the remaining two targets. All other Ss pointed to all targets an equal number of times (13 times each). Total number of trials in each group was the same (39). In addition, half of the Ss in each condition were allowed to observe their self-initiated and controlled arm movements during the exposure condition by placing their fingers in the aluminum container on the top level of the wooden box. The remainder of the Ss simply observed targets under 20-diopter prismatic displacement, but were not able to observe movements, since their arms were placed in the lower level of the wooden box. S observed her self-initiated and controlled hand move laterally across her visual field during the exposure condition one cycle every $6 \mathrm{sec}$. A metronome was used to indicate this rate. Furthermore, base shift of prisms was counterbalanced across all experimental conditions. In the postexposure test, which immediately followed the exposure condition. all Ss in all conditions were required to point five times each, in a random order, at each of the three vertical black lines centered on the tagboard on the back of the apparatus. As in the preexposure condition, S's hand was not visible to her.

\section{RESULTS}

The dependent variable in this study was the difference between S's judgments of target location in the pre- and postexposure conditions. If the difference deviated from the actual location of targets in the direction opposite of that of the prismatic displacement, it was referred to as a negative aftereffect; this was the measure of visual adaptation in the present study.

A mixed design analysis of variance was performed with three between-S variables-differential accentuation via frequency of preexposure pointing to targets (DA), unobserved or observed arm movement ( $U$ or $O$ ), and right or left prismatic shift (RL)-and one within-S variable-location of targets in the visual field, whether left, center, or right (LCR). No significant difference, $F(1,40)=2.84, p<.10$, in mean adaptation to visual displacement was found as a function of whether the prism shift was to the left or right. In addition, no significant difference, $F(1,40)=2.06$, in mean adaptation was found as a function of differential accentuation (DA).

Two-tailed $t$ tests for correlated measures between the pre- and postexposure measures of target location were used to test for significant negative aftereffects from zero. When Ss were required to point 13 times to each of the three targets during the preexposure manipulation, a mean of $4.2 \mathrm{deg}$ adaptation was found, $\mathrm{t}(23)=7.30$,

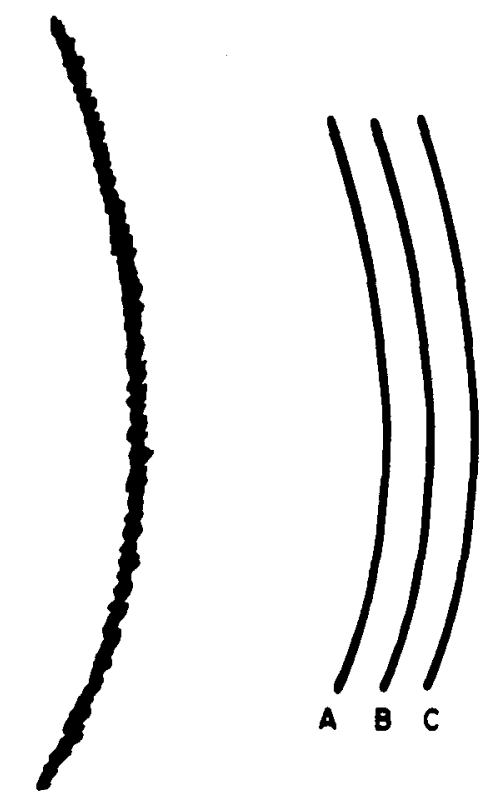

\section{EDGES OF VISUAL FIELD BASE LEFT PRISM SHIFT}

Fig. 3. S's view of a 33-cm-wide visual field with three visual targets present during 20-diopter prismatic displacement, base left. Target $C$ forms the boundary of the visual field on the right (most peripheral target), and Target $A$ is the extreme nonboundary and most centrally located target during prism exposure.

$p<.001$. For Ss required to point 29 times to the extreme nonboundary target (most centrally located target during prism exposure) and five times to each of the remaining two targets in the visual field, a mean of $3.0 \mathrm{deg}$ was found, $\mathfrak{t}(23)=4.68, \mathrm{p}<.001$.

The difference in resultant adaptation as a function of whether Ss were or were not allowed to observe their arm movements during the exposure condition ( $\mathrm{U}$ or $\mathrm{O}$ ) was not significant, $F(1,40)=0.23$. In addition, subsequent Newman-Keuls analysis showed no significant difference between any of the conditions in the (DA) by (U or $\mathrm{O}$ ) interaction. Interactions between other between-S variables were not found to be significant.

The interaction of DA by Individual Targets in the Visual Field (LCR) by Base Shift of Prisms (RL) was found to be significant, $F(2,80)=57.90, p<.001$. Thus, whenthe visual field was shifted to the left of straight ahead during the exposure condition and all targets received an equal number of pointing trials in the preexposure test (13), the greatest amount of resultant adaptation occurred for the left test target $(4.5 \mathrm{deg})$. For base left prisms, the greatest mean adaptation was found for the right target $(6.3 \mathrm{deg})$-see Fig. 4 . These results 


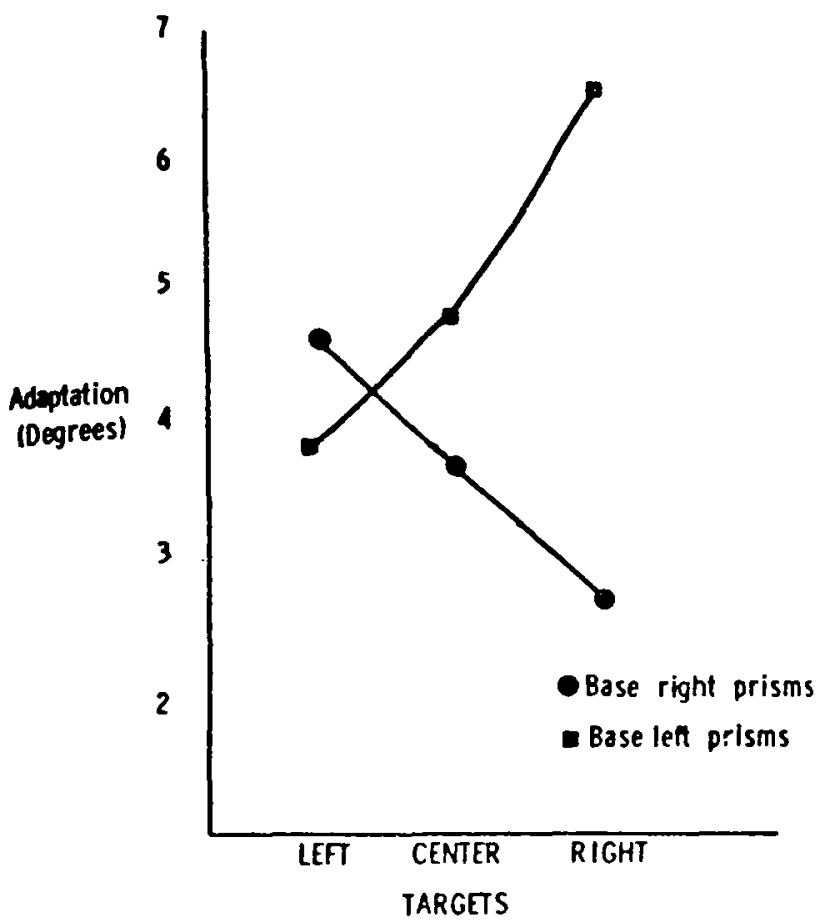

Fig. 4. Mean amount of adaptation for three test targets as a function of prismatic shift and all targets in visual field receiving 13 pointing trials each during the preexposure test.

are comparable to those found by Melamed. Halay, and Gildow (1973).

When the visual field was shifted to the left of straight ahead in the exposure condition and the most central target during prism exposure (right target) received 29 pointing trials in the preexposure test while the remaining two targets received five trials each, adaptation was found to be greatest for the target receiving the most pointing trials in the preexposure test $(4.7 \mathrm{deg})$. For base left prisms, where the left nonboundary and most centrally located target during prism exposure received 29 pointing trials in the preexposure test and the reniainin $\tilde{E}$ iwo targets received five trials each. again the target receiving the largest number of pointing trials in the preexposure condition showed the greatest magnitude of adaptation $(5.2 \mathrm{deg}$, see Fig. 5). Significant adaptation to all targets, regardless of base shift, was found in the 13-13-13 condition. In the 29-5-5 condition, significant adaptation was found for all targets except for the left with base right prisms (the most peripheral target: see Table 1). The DA by LCR by RL by UO interaction was not found to be significant, $F(2.80)=0.68$.

Differential adaptation, as measured by targets in the 13.13-13 condition. was found between the left, central target $(3.8 \mathrm{deg})$ and the right. peripheral target $(6.3 \mathrm{deg})$ with base left prisms, Newman-Keuls. $p<.01$. A significant difference was also found between these two targets $(4.5 \mathrm{deg}$ for the peripheral. left target vs $2.5 \mathrm{deg}$ for the right. central target) for base right prisms. Newman-Keuls. $p<.01$.
The comparison of magnitude of adaptation across targets as a function of prismatic shift of the visual field in the 29-5-5 condition (see Table 1) showed a significant difference between the left, central target $(5.2 \mathrm{deg})$ and the right, peripheral target $(2.3 \mathrm{deg})$ for base left prisms, Newman-Keuls, $p<.01$, and for base right prisms $(0.6 \mathrm{deg}$ for the peripheral, left target vs $4.7 \mathrm{deg}$ for the right, central target, Newman-Keuls, $\mathrm{p}<.01$ ).

\section{DISCUSSION}

The results of the present study have confirmed previous research in showing that if the frequency of preexposure pointing was held constant across target location, the greatest adaptation occurred for the target that, when prismatically shifted, was the most peripheral (Wallace, 1972; Melamed et al, 1973). Thus, when Ss point equally to targets present in a preexposure condition, the kinesthetic and proprioceptive feedback with respect to their location in regard to body posture is approximately equal provided that the arrangement of the stimuli is symmetrical in S's field of view. This was the case in the present study and in the previously cited research. Harris (1965), as discussed in the introduction, has suggested that adaptation results from proprioceptive sources of feedback concerning arm location with regard to body position being compared to visual sources of information concerning arm location. The perceived discrepancies in the aforementioned sources of information result in adaptation during prism exposure. The most peripherally shifted target, since $S$

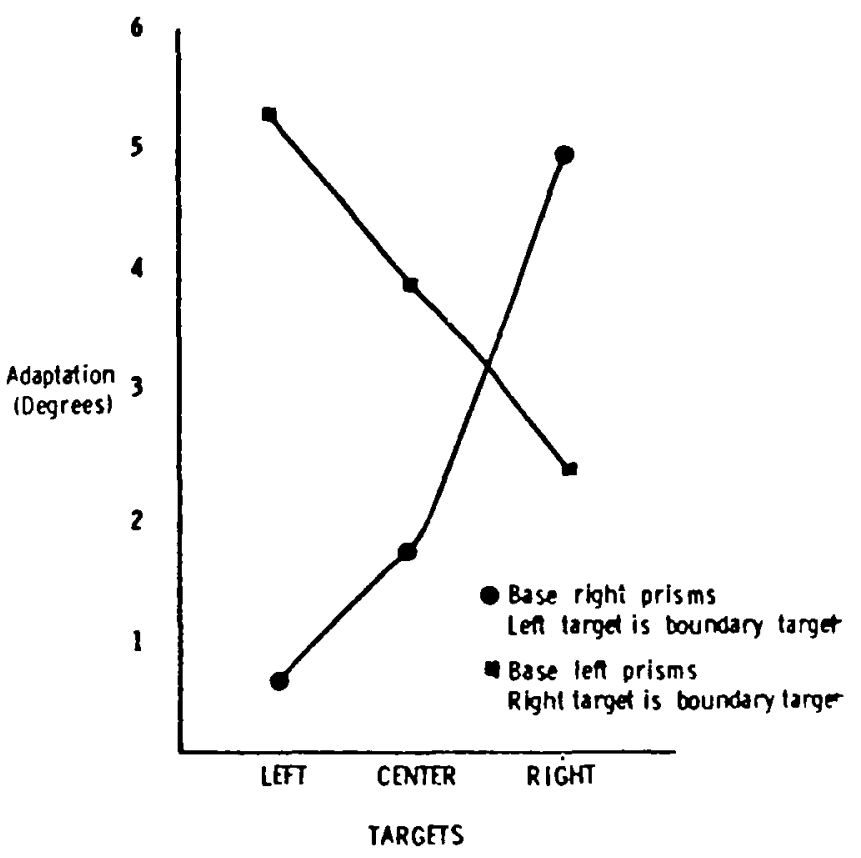

Fig. 5. Mean amount of adaptation as a function of prismatic shift and the extreme nonboundary target receiving 29 pointing trials during the preexposure test. with remaining targets receiving 5 trials each in the preexposure test. 
initiates the greatest amplitude movements with respect to that target compared to the other two targets, would result in greater adaptation as a result of the greater perceived discrepancies.

The present study also suggests that exposure sources of visual and proprioceptive information concerning arm location with regard to target location and body position are compared with the S's possible memory of preexposure proprioception if $S$ points more often to one target compared to the other two targets during the preexposure procedure. In such a situation, more kinesthetic and proprioceptive information becomes available concerning arm location with regard to the most frequently pointed-to target compared to the other two. Thus, S's memory of the preexposure kinesthetic feedback would be greatest in the present study for the target that, when prismatically shifted, became the most central target. In this case, the perceived discrepancy between preexposure and exposure kinesthetic feedback would be greatest for the more central target, and the adaptation of the kinesthetic feedback would also be expected to be greater for this target. Thus, in the present experiment, the greatest adaptation was seen for the target that was given the greatest frequency of preexposure pointing, the most central target; if preexposure pointing frequency had been equal, the greatest adaptation would have been expected for the target shifted to the periphery.

It is interesting to note that differential pointing practice in the preexposure condition (see Fig. 5) reversed the slopes of the curves under equal pointing practice (Fig. 4). Although it might be expected that adaptation magnitude would be large for both the target $S$ points to most frequently and the peripheral target producing a V-shaped function, this was not the case. Thus, differential preexposure pointing practice served to reduce the magnitude of the discrepancy between the visual and the proprioceptive location of S's arm with respect to peripheral target location and body position. This, in turn, reduced the magnitude of adaptation to the peripherally located target. A possible explanation for this reduction in adaptation magnitude may relate to the sources of proprioceptive information available to $S$ concerning arm location in a differential preexposure pointing task compared to an equal preexposure pointing task. Requiring differential pointing practice may make proprioceptive feedback concerning arm location with respect to a specific target location nore salient as a source of information for locating a arget position than proprioceptive feedback from amplitude of arm movement when reaching for a target location (e.g., central vs peripheral). In such a situation, greater adaptation would be expected for the target $S$ points to most frequently compared to the target $S$ initiates the greatest amplitude of movement towards (e.g., the peripheral target). The results of the present study show this to be the case.

The present study thus shows that the visual and
Table 1

Mean Degrees Adaptation for Three Test Targets as a Function of Differential Accentuation to Targets in the Preexposure Condition

\begin{tabular}{|c|c|c|c|c|c|c|}
\hline & \multicolumn{2}{|c|}{$\begin{array}{c}\text { Adaptation } \\
\text { for Left } \\
\text { Target } \\
\end{array}$} & \multicolumn{2}{|c|}{$\begin{array}{c}\text { Adaptation } \\
\text { for Center } \\
\text { Target }\end{array}$} & \multicolumn{2}{|c|}{$\begin{array}{c}\text { Adaptation } \\
\text { for Right } \\
\text { Target }\end{array}$} \\
\hline & $\begin{array}{c}\text { Mean } \\
\text { Deg }\end{array}$ & SD & $\begin{array}{c}\text { Mean } \\
\text { Deg }\end{array}$ & SD & $\begin{array}{c}\text { Mean } \\
\text { Deg }\end{array}$ & SD \\
\hline $\begin{array}{l}13-13-13^{* *} \\
\text { Base Right } \\
\text { Base Left }\end{array}$ & $\begin{array}{l}4.5^{*} \\
3.8^{*}\end{array}$ & $\begin{array}{l}1.71 \\
1.48\end{array}$ & $\begin{array}{l}3.5^{*} \\
4.8^{*}\end{array}$ & $\begin{array}{l}1.58 \\
1.40\end{array}$ & $\begin{array}{l}2.5^{*} \\
6.3^{*}\end{array}$ & $\begin{array}{l}1.04 \\
1.89\end{array}$ \\
\hline $\begin{array}{l}29-5-5 \dagger \\
\text { Base Right } \\
\text { Base Left }\end{array}$ & $\begin{array}{l}0.6 \\
5.2^{*}\end{array}$ & $\begin{array}{l}0.37 \\
2.11\end{array}$ & $\begin{array}{l}1.8^{*} \\
3.7^{*}\end{array}$ & $\begin{array}{l}1.07 \\
1.82\end{array}$ & $\begin{array}{l}4.7^{*} \\
2.3^{*}\end{array}$ & $\begin{array}{l}1.75 \\
1.15\end{array}$ \\
\hline
\end{tabular}

${ }^{*} p<.01$; analyses made by two-tailed $t$ tests for correlated measures with $d f=11$.

${ }^{*}$ * Ss were asked to point to each target 13 times during the preexposure test.

fFor base right prisms, the right target received 29 pointing trials in the preexposure test; for base left prisms, the left target received 29 trials in the preexposure test. Remaining targets received five trials each.

proprioceptive information for various targets can be manipulated both by target location and by frequency of pointing to the target in the preexposure period. The results of the manipulation can then be measured in terms of the degree of adaptation produced for that target. The present study suggests that consciousness is not an important part of this process, since the DA by LCR by RL interaction was not significantly different for Ss permitted arm observation during prism exposure and those not allowed to view arm movement. Conscious correction should be able to play a larger role in adaptation when $S$ could observe his arm movement during prism exposure, but the lack of significance of the above three-way interaction between Ss allowed arm observation and those not allowed such observation during prism exposure indicated that the presence or absence of observation had little effect on the differential adaptation.

\section{REFERENCES}

Harris, C. S. Perceptual adaptation to inverted, reversed, and displaced vision. Psychological Review, 1965, 72, 419-444.

Melamed, L. E., Halay, M., \& Gildow, J. An examination of the role of task oriented attention in the use of active and passive movement in visual adaptation. Journal of Experimental Psychology, 1973, 98, 125-130.

Rock, I. The nature of perceptual adaptation. New York: Basic Books, 1966.

Wallace, B. An examination of the role of attention and visual-proprioceptive information on visual adaptation to prismatic displacement. Unpublished doctoral dissertation, Kent State University, 1972.

(Received for publication March 16. 1973; revision received July 13.1973 .) 